\title{
Spontaneously modulated spin textures in a dipolar spinor Bose-Einstein condensate
}

\author{
M. Vengalattore ${ }^{1}$, S. R. Leslie ${ }^{1}$, J. Guzman ${ }^{1}$ and D. M. Stamper-Kurn ${ }^{1,2}$ \\ ${ }^{1}$ Department of Physics, University of California, Berkeley CA 94720 \\ ${ }^{2}$ Materials Sciences Division, Lawrence Berkeley National Laboratory, Berkeley, CA 94720
}

(Dated: November 21, 2018)

\begin{abstract}
Helical spin textures in a ${ }^{87} \mathrm{Rb} F=1$ spinor Bose-Einstein condensate are found to decay spontaneously toward a spatially modulated structure of spin domains. This evolution is ascribed to magnetic dipolar interactions that energetically favor the short-wavelength domains over the longwavelength spin helix. This is confirmed by eliminating the dipolar interactions by a sequence of $\mathrm{rf}$ pulses and observing a suppression of the formation of the short-range domains. This study confirms the significance of magnetic dipole interactions in degenerate ${ }^{87} \mathrm{Rb} F=1$ spinor gases.
\end{abstract}

In a wide range of materials, the competition between short- and long-range interactions leads to a rich landscape of spatially modulated phases arising both in equilibrium and as instabilities in non-equilibrated systems 1, 2]. In classically ordered systems such as magnetic thin films [3] and ferrofluids [4], short-range ferromagnetic interactions are commonly frustrated by the longrange, anisotropic magnetic dipolar interaction, rendering homogeneously magnetized systems intrinsically unstable to various morphologies of magnetic domains [5]. Long-range interactions are also key ingredients in many models of strongly correlated electronic systems [6] and frustrated quantum magnets 7 .

In light of their relevance in materials science, strong dipole interactions have been discussed as an important tool for studies of many-body physics using quantum gases of atoms and molecules, offering the means for quantum computation [8], simulations of quantum magnetism [9] and the realization of supersolid or crystalline quantum phases [10, 11]. However, in most ultracold atomic gases, the magnetic dipolar interaction is dwarfed by the contact ( $s$-wave) interaction. Hence, experimental efforts to attain dipolar quantum gases have focused on non-alkali atoms, notably ${ }^{52} \mathrm{Cr}$ with its large magnetic moment [12], and on polar molecules [13].

In this Letter, we demonstrate that magnetic dipole interactions play a critical role in the behaviour of a quantum degenerate $F=1$ spinor Bose gas of ${ }^{87} \mathrm{Rb}$. In this quantum fluid, $s$-wave collisions yield both a spin-independent and a spin-dependent contact interaction [14, 15, 16], with strengths proportional to $\bar{a}=$ $\left(2 a_{2}+a_{0}\right) / 3$ and $\Delta a=\left(a_{2}-a_{0}\right) / 3$, respectively, where the scattering length $a_{F}$ describes collisions between particles of total spin $F$. In ${ }^{87} \mathrm{Rb}$, with $a_{0}\left(a_{2}\right)=5.39(5.31)$ $\mathrm{nm}$, the spin-dependent contact interaction is far weaker than the spin-independent one; nevertheless, it is a critical determinant of the magnetic properties of degenerate $F=1{ }^{87} \mathrm{Rb}$ gases [17, 18, 19]. The magnetic dipole interaction strength may be parameterized similarly by a length $a_{d}=\mu_{0} g_{F}^{2} \mu_{B}^{2} m /\left(12 \pi \hbar^{2}\right)$, where $\mu_{0}$ is the permeability of vacuum, $g_{F}=1 / 2$ the gyromagnetic ratio, $\mu_{B}$ the Bohr magneton and $m$ the atomic mass [20]. Given $a_{d} / \Delta a=0.4$, the $F=1$ spinor Bose gas of ${ }^{87} \mathrm{Rb}$ is an essentially dipolar quantum fluid [21].

In our experiment, the influence of dipolar interactions on the spinor gas is evidenced by the spontaneous dissolution of deliberately imposed long-wavelength helical spin textures, in favor of a finely modulated pattern of spin domains. We ascribe the emergence of this modulated phase to the magnetic dipole energy that disfavors the homogenously magnetized state and drives the fluid toward short-wavelength spin textures. To test this ascription, we re-examine the behavior of spin helices in condensates in which the dipolar interaction is eliminated using a rapid sequence of $\mathrm{rf}$ pulses. The suppression of the modulated phase observed in this case confirms the crucial role of dipolar interactions.

For this work, spin-polarized ${ }^{87} \mathrm{Rb}$ condensates of up to $2.3(1) \times 10^{6}$ atoms in the $\left|F=1, m_{F}=-1\right\rangle$ hyperfine state and at a kinetic temperature of $T \simeq 50 \mathrm{nK}$ were confined in a single-beam optical dipole trap characterized by trap frequencies $\left(\omega_{x}, \omega_{y}, \omega_{z}\right)=2 \pi \times(39,440,4.2)$ $\mathrm{s}^{-1}$. The Thomas-Fermi condensate radius in the $\hat{y}$ (vertical) direction $\left(r_{y}=1.8 \mu \mathrm{m}\right)$ was less than the spin healing length $\xi_{S}=\left(8 \pi \Delta a n_{0}\right)^{-1 / 2}=2.4 \mu \mathrm{m}$ where $n_{0}=2.3 \times 10^{14} \mathrm{~cm}^{-3}$ is the peak density of the condensate. This results in a spinor gas that is effectively two-dimensional with regard to spin dynamics.

The condensate was transversely magnetized by applying a $\pi / 2 \mathrm{rf}$ pulse in the presence of an ambient magnetic field of $B_{0}=165(5) \mathrm{mG}$ aligned to the $\hat{z}$ axis. Stray magnetic gradients (curvatures) were canceled to less than $0.14 \mathrm{mG} / \mathrm{cm}\left(4.3 \mathrm{mG} / \mathrm{cm}^{2}\right)$. A helical spin texture was then prepared by applying a transient magnetic field gradient $d B_{z} / d z$ for a period $\tau_{p}=5-8 \mathrm{~ms}$. Larmor precession of the atomic spins in this inhomogeneous field resulted in a spatial spin texture with a local dimensionless spin of $\mathbf{F}=\cos \left(\kappa z+\omega_{L} t\right) \hat{x}+\sin \left(\kappa z+\omega_{L} t\right) \hat{y}$, where $\vec{\kappa}=\left(g_{F} \mu_{B} / \hbar\right)\left(d B_{z} / d z\right) \tau_{p} \hat{z}$ is the helix wavevector. The fast time variation at the $\omega_{L} / 2 \pi \simeq 115 \mathrm{kHz}$ Larmor precession frequency will be henceforth ignored by considering the spin at a particular instant in this rapid evolution. The helix pitch $\lambda=2 \pi / \kappa$ ranged between 50 and $150 \mu \mathrm{m}$. Given $\lambda \gg \xi_{S}$, the kinetic energy per atom in this spin texture, $E_{\kappa}=\hbar^{2} \kappa^{2} / 4 m$, was always negligible compared to the ferromagnetic contact interaction energy [22]. 


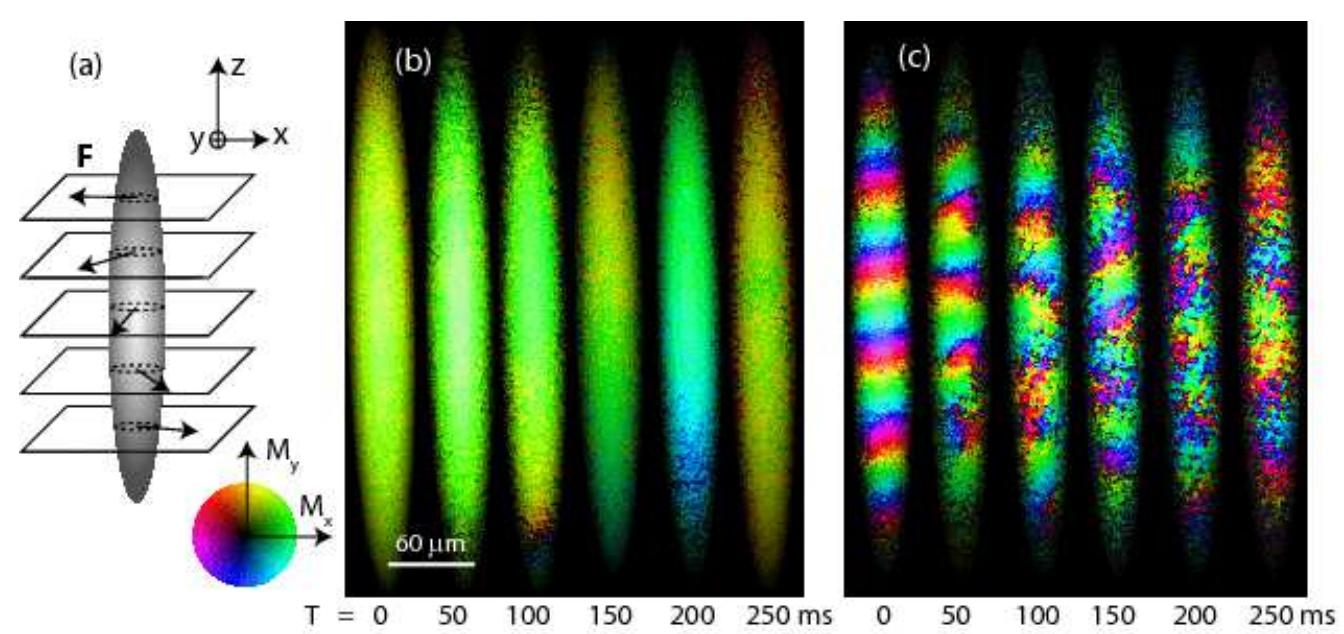

FIG. 1: Spontaneous dissolution of helical textures in a quantum degenerate ${ }^{87} \mathrm{Rb}$ spinor Bose gas. A transient magnetic field gradient is used to prepare transversely magnetized (b) uniform or (a, c) helical magnetization textures. The transverse magnetization column density after a variable time $T$ of free evolution is shown in the imaged $x-z$ plane, with orientation indicated by hue and amplitude by brightness (color wheel shown). (b) A uniform texture remains homogeneous for long evolution times, while (c) a helical texture with pitch $\lambda=60 \mu \mathrm{m}$ dissolves over $\sim 200 \mathrm{~ms}$, evolving into a sharply spatially modulated texture.

The helical spin texture was then allowed to evolve in a homogenous magnetic field for a variable time before the vector magnetization was measured using a sequence of non-destructive phase contrast images. Because of Larmor precession, a rapid sequence of images taken with circularly polarized light propagating along the $\hat{y}$ direction can be analyzed to determine the column-integrated magnetization perpendicular to the ambient field [23, 24], with vector components $\tilde{M}_{x, y}=\left(g_{F} \mu_{B}\right) \tilde{n} F_{x, y}$ where $\tilde{n}$ the column number density. Subsequent to this imaging sequence, a $\pi / 2$ pulse was applied to rotate the longitudinal spin $F_{z}$ into the transverse spin plane, and a second sequence of images was obtained. A least-squares algorithm comparing data from the two imaging sequences allowed the longitudinal magnetization $\tilde{M}_{z}$ to be determined [25].

The evolution of helical spin textures is portrayed in Fig. 1. While uniform spin textures $\left(\lambda \gg 2 r_{z}\right)$ remained homogenous for long times, helical textures $\left(\lambda<2 r_{z}\right)$ spontaneously develop short-wavelength modulations of the magnetization. This modulated phase is characterized by spin domains with typical dimensions of $\simeq 10 \mu \mathrm{m}$, much smaller than the pitch of the imprinted helix, with the magnetization varying sharply between adjacent domains. The spatial modulations nucleated in regions that varied from shot to shot but gradually grew to encompass the entire condensate.

To quantify this behaviour, we considered the power spectrum of the spatial Fourier transform of the vector magnetization, $\left|\tilde{\mathbf{M}}\left(k_{x}, k_{z}\right)\right|^{2}$, where $\left(k_{x}, k_{z}\right)$ is the spatial wavevector in the image plane. This spectrum was found to consist of two distinct components: a central component that quantifies the long-range order of
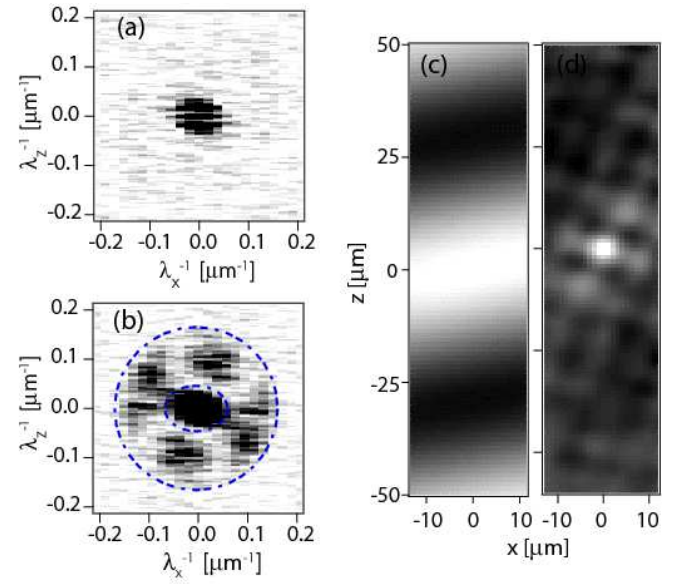

FIG. 2: Power spectrum of the spatial Fourier transform and the two-point correlation function $G(x, z)$ for the initial spin helix (a, c) and the spontaneously modulated phase (b, d). These data are derived from the same image sequence shown in Fig. 1 (d). The images (a, c) correspond to an evolution time $T=0 \mathrm{~ms}$ while (b, d) correspond to an evolution time $T=250 \mathrm{~ms}$. The short-range spatial order is defined as the integrated spectral power in the annular region shown in (b).

the helical texture, and a second concentration of spectral power at a discrete set of wavevectors of magnitude $k_{\text {mod }} \simeq 2 \pi /(10 \mu \mathrm{m})$ representing the short-range order of the final modulated texture. After subtracting out the background representing image noise, we divided spatial Fourier space into regions indicated in Fig. 2 and defined the integrated spectral power in the central region (annular region) as the parametrization of long-range (shortrange) spatial order in the quantum fluid. 


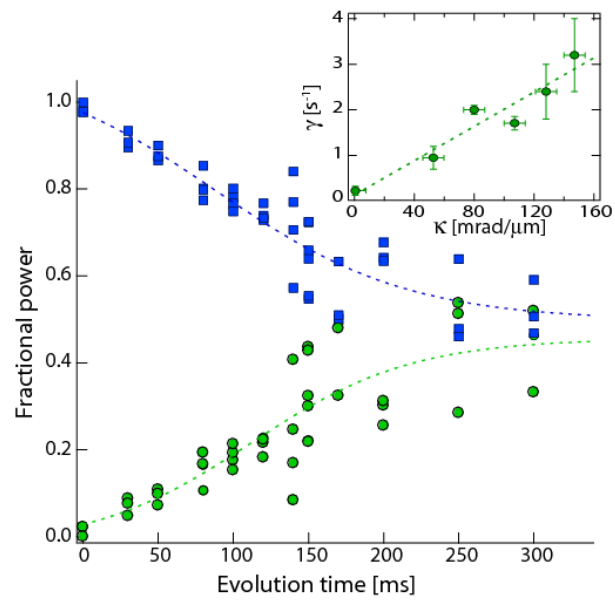

FIG. 3: Growth of the spontaneously modulated phase $(\bullet)$ coincides with a reduction in the integrated energy in the low spatial frequency region $(\boldsymbol{\square})$. The data shown correspond to an initial helical pitch of $60 \mu \mathrm{m}$. Inset: The initial growth rate $\gamma$ of the modulated phase as a function of the helix wavevector. These were extracted from linear fits of the short-range order parameter at short evolution times.

The formation of the spontaneously modulated texture is reflected in the reduction of the long-range order parameter and the concomitant rise of the short-range order parameter (Fig. 3). During this process, the total spectral power was found to be roughly constant indicating that the bulk of the quantum fluid remains fully magnetized even as the long-range order is reduced. The growth rate $\gamma$ of the short-range order parameter determined from such data was found to rise monotonically with the wavevector $\kappa$ of the initial helical texture. While the long-range order was found to decrease after sufficiently long evolution times even in condensates prepared with nearly uniform magnetization, we note that stray magnetic field inhomogeneities of $\sim 5 \mu \mathrm{G}$ across the axial length of the condensate would by themselves produce a helical winding across the condensate over a period of $300 \mathrm{~ms}$, constraining our ability to test the stability of homogenous spin textures.

Another measure of the spontaneous short-range modulation in the condensate is the appearance of polar-core spin vortices throughout the gas. Such vortices were identified as in Ref. [19] by a net winding of the transverse magnetization along a closed two-dimensional path of non-zero magnetization in the imaged gas. The number of identified spin vortices was roughly proportional to the short-range order parameter, with no vortices identified in the initially prepared spin helix and up to 6 vortices/image identified in the strongly modulated texture produced after free evolution. In each instance, the number of vortices with positive and negative charge was found to be approximately equal.

A striking feature in the evolution of spin textures is the significant rise in the kinetic energy of the condensed atoms, reaching a value of $\hbar^{2} k_{\text {mod }}^{2} / 8 m=h \times 6 \mathrm{~Hz}$ per atom given that roughly half the spectral weight of the texture's magnetization is at the wavevector $k_{\text {mod }}$. One expects the total energy per atom in the condensate to be constant during this evolution, or even to diminish through the transfer of energy to the non-condensed portion of the gas. Yet, in examining the energy of the initially prepared spin helix, we find the local contactinteraction energy is minimized, the quadratic Zeeman energy is just $q / 2=h \times 1 \mathrm{~Hz}$ at the ambient magnetic field, and the kinetic energy of the spin helix is just $E_{\kappa} / 2<h \times 0.5 \mathrm{~Hz}$ for a helix pitch of $\lambda>50 \mu \mathrm{m}$.

This apparent energetic deficit of the spin helix state can be accounted for by the magnetic dipole interaction. The on-axis magnetic field produced by a spin helix in an infinite axial column of gas with a gaussian transverse density profile can be simply calculated. From this calculation, we estimate that a gas with uniform transverse magnetization possesses an excess of $E_{d}=\mu_{0} g_{F}^{2} \mu_{B}^{2} n_{0} / 2 \sim h \times 5 \mathrm{~Hz}$ compared to the energy of a tightly wound helix, a figure that closely matches the excess kinetic energy of the finely modulated texture.

To confirm the role of magnetic dipolar interactions in the evolution of these spin textures, we employed a modification of the NMR technique of spin-flip narrowing 26] to eliminate effectively the dipolar interactions. The interaction energy of two magnetic dipoles separated by the displacement vector $\mathbf{r}$ is proportional to $\mathbf{F}_{1} \cdot \mathbf{F}_{2}-3\left(\hat{r} \cdot \mathbf{F}_{1}\right)\left(\hat{r} \cdot \mathbf{F}_{2}\right)$. If both dipoles experience rapid, common rotations that evenly sample the entire $S O(3)$ group of rotations, the interaction energy will average to zero regardless of the relative orientations of the spin vectors $\mathbf{F}_{1,2}$ and of the displacement vector $\mathbf{r}$. We note that such spin rotations also annul the quadratic Zeeman shift. However, since the character of the spontaneously modulated phase was observed to be unchanged as $q$ was varied over a factor of $5\left(0.4<\frac{1}{h} q / 2<2 \mathrm{~Hz}\right)$, we ignore this small difference.

Experimentally, after the initial spin texture had been prepared as before, we effected such spin rotations by applying a rapid sequence of $\pi / 2 \mathrm{rf}$ pulses to the Larmor precessing atoms at random intervals, and, thus, along random rotation axes, at a mean rate of $1-2 \mathrm{kHz}$. The spin helix was allowed to evolve under the constant action of these dipole-cancelation pulses until the pulses ceased and the sample was imaged as described earlier. As shown in Fig. 4, in the absence of dipole interactions a spin helix was still prone to spontaneous spatial modulation, consistent with the predictions of dynamic instabilities made in Refs. 27] and [28]. However, the depth of this modulation was significantly suppressed, as seen from the smaller spectral weight of short-range magnetic order. This suppression was less evident for large helix wavevectors $(\kappa>110 \mathrm{mrad} / \mu \mathrm{m})$, presumably due to the faster growth of the spontaneously modulated phase. 

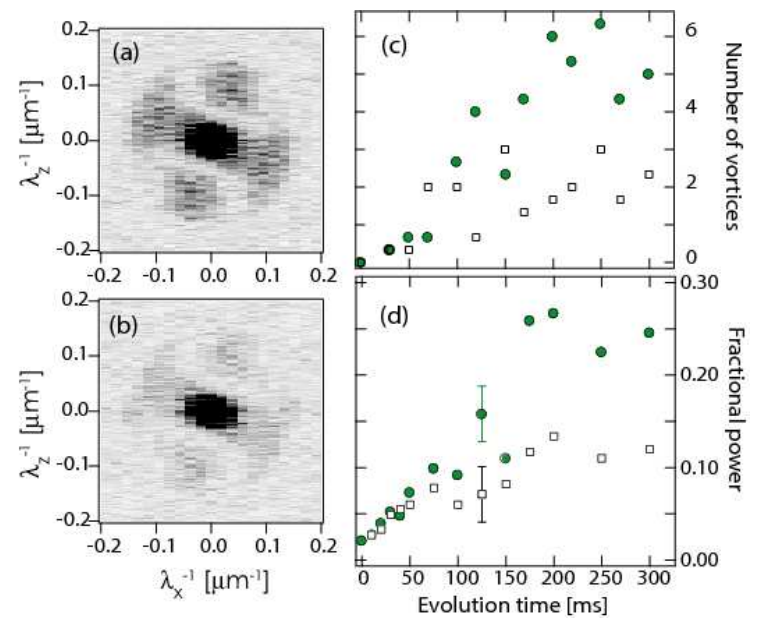

FIG. 4: Spatial power spectra (a) without or (b) with the application of rapid $\mathrm{rf}$ pulses during the $200 \mathrm{~ms}$ evolution following the preparation of the helical spin texture, are averaged over four experimental repetitions. Eliminating the dipolar interactions suppresses the short-range spatial modulation of the final spin texture. (c) The average number of detected spin vortices and (d) the short-range order parameter are shown vs. evolution time. Square symbols indicate data obtained when the dipolar interaction is spatially averaged. In all cases, the initial helix pitch was $\lambda=80 \mu \mathrm{m}$.

The reduction of the excess kinetic energy of the final spin texture in the spin-rotation-averaged sample supports our identification of the dipole energy as its source.

Finally, we note the distinct six-fold structure in the spatial Fourier spectrum of the spontaneously modulated phase (Fig. 2(b)). The interpretation of this structure is aided by considering the spatial correlation function of the magnetization, which we define as

$$
G(\delta \mathbf{r})=\frac{\sum_{\mathbf{r}} \tilde{\mathbf{M}}(\mathbf{r}+\delta \mathbf{r}) \cdot \tilde{\mathbf{M}}(\mathbf{r})}{\left(g_{F} \mu_{B}\right)^{2} \sum_{\mathbf{r}} \tilde{n}(\mathbf{r}+\delta \mathbf{r}) \tilde{n}(\mathbf{r})}
$$

For the initial spin helix, this correlation function shows the long-range sinusoidal variation of the transverse magnetization at the corresponding pitch. In contrast, the final texture shows significant short-range correlations in both the $\hat{x}$ and $\hat{z}$ directions, with regions of opposite alignment arranged in the form of a checkerboard with a lattice spacing $l_{m} \simeq 10 \mu \mathrm{m}$ (Fig. 2(d)). While these correlations are strongest at short range, they persist, albeit with diminished strength, even for separations $\delta r \gg l_{m}$. This lattice structure is suppressed in helices evolving under the active cancelation of dipolar interactions.

In conclusion, we observe a magnetic-dipole-mediated instability resulting in the emergence of spontaneously ordered spin domains in a quantum degenerate spinor Bose gas. The demonstration of the significance of magnetic dipolar interactions in a ${ }^{87} \mathrm{Rb}$ spinor gas presents a new arena for the study of dipolar quantum gases. Of particular interest is the influence of such anisotropic interactions on the true ground state of these spinor gases and the question of whether the self-organized modulated spin texture represents an unforeseen equilibrium phase of this dipolar quantum fluid.

We acknowledge insightful discussions with E. Demler, C. H. Greene and A. Lamacraft. This work was supported by the NSF, the David and Lucile Packard Foundation, and DARPA's OLE program. Partial personnel and equipment support was provided by the Division of Materials Sciences and Engineering, Office of Basic Energy Sciences. S. R. L. acknowledges support from the NSERC.

[1] M. C. Cross and P. C. Hohenberg, Rev. Mod. Phys. 65, 851 (1993).

[2] M. Seul and D. Andelman, Science 267, 476 (1995).

[3] T. Garel and S. Doniach, Phys. Rev. B 26, 325 (1982).

[4] S. Odenbach, ed., Ferrofluids: magnetically controllable fluids and their applications, vol. 594 of Lecture Notes in Physics (Springer, New York, 2002).

[5] K. De'Bell, A. B. MacIsaac and J. P. Whitehead, Rev. Mod. Phys. 72, 225 (2000).

[6] E. Dagotto, Science 309, 257 (2005).

[7] U. Schollwöck et al., eds., Quantum Magnetism, vol. 645 of Lecture Notes in Physics (Springer, Berlin, 2004).

[8] D. Jaksch et al., Phys. Rev. Lett. 85, 2208 (2000).

[9] A. Micheli, G. K. Brennan and P. Zoller, Nature Physics 2, 341 (2006).

[10] K. Góral, L. Santos and M. Lewenstein, Phys. Rev. Lett. 88, 170406 (2002).

[11] H. P. Büchler et al., Phys. Rev. Lett. 98, 060404 (2007).

[12] T. Lahaye et al., Nature 448, 672 (2007).

[13] J. Doyle et al., Eur. Phys. J D 31, 149 (2004).

[14] T. L. Ho, Phys. Rev. Lett. 81, 742 (1998).

[15] T. Ohmi and K. Machida, J. Phys. Soc. Jpn. 67, 1822 (1998).

[16] J. Stenger et al., Nature 396, 345 (1998).

[17] M. -S. Chang et al., Nature Physics 1, 111 (2005).

[18] J. Kronjager et al., Phys. Rev. Lett. 97, 110404 (2006).

[19] L. Sadler et al., Nature 443, 312 (2006).

[20] L. Santos, G. Shlyapnikov and M. Lewenstein, Phys. Rev. Lett. 90, 250403 (2003).

[21] S. Yi and H. Pu, Phys. Rev. Lett. 97, 020401 (2006).

[22] In a transversely magnetized spin texture in an $F=1$ spinor condensate, only half of the atoms, those in the $m_{F}= \pm 1$ magnetic sublevels, acquire kinetic energy.

[23] M. Vengalattore et al., Phys. Rev. Lett. 98, 200801 (2007).

[24] J. M. Higbie et al., Phys. Rev. Lett. 95, 050401 (2005).

[25] Further details on the imaging system and signal analysis will be discussed elsewhere.

[26] C. Slichter, Principles of Magnetic Resonance (Springer, New York, 1978).

[27] A. Lamacraft, preprint, arXiv:0710.1848

[28] R. W. Cherng et al., preprint, arXiv:0710.2499. 